\title{
Influence of Tensioner's Mobilization on the Centralization of Symptoms in Cervicobrachial Pain Syndrome: A Randomized Controlled Trial
}

\author{
Kavita Sudhakar ${ }^{1}$, Sohrab A. Khan ${ }^{2}$, Avi Saraswat ${ }^{3}$, Meena Makhija ${ }^{4}$ \\ ${ }^{1}$ Department of Physiotherapy, Mother Teresa Saket College of Physiotherapy, Chandimandir, India \\ ${ }^{2}$ Department of Physiotherapy, Jamia Hamdard, New Delhi, India \\ ${ }^{3}$ Department of Physiotherapy, Abhinav Bindra Targeting Performance, Mohali, India \\ ${ }^{4}$ Department of Physiotherapy, Indian Spinal Injuries Centre-Institute of Rehabilitation Sciences, New Delhi, India
}

\begin{abstract}
Study Design: This study was designed as a randomized controlled trial.
Purpose: The present study aimed to determine the impact of neural mobilization by tensioner's technique (NMTT) on the centralization of symptoms and pain in patients with cervicobrachial pain syndrome (CBPS).

Overview of Literature: CBPS is a disabling condition of the neck that is characterized by pain and paresthesia in the upper quarter. Several techniques have successfully provided immediate and long-term relief in CBPS; however, few studies have evaluated the effect of these techniques on the centralization of symptoms.

Methods: Thirty patients aged 18-45 years with a complaint of pain in the neck that had persisted for 2-12 weeks radiating to the arm and fulfilling Elvey's criteria were randomly selected and divided into two groups. Group A received NMTT plus conventional treatment (hot pack and postural advice with cervical lateral glide), and group B received only conventional treatment 3 times a week for 2 weeks. The outcome measures were Wernicke's scale score for the centralization of symptoms and Visual Analog Scale score for pain intensity. Within- and between-group comparisons were made before initiating treatment and at the end of the 3rd and 6th sessions. Within group analyses for the centralization values were performed using Friedmann test, and between-group analyses were performed using Mann-Whitney test. A 2×3 mixed model of the analysis of variance was used for analyzing the pain levels.

Results: There was a significant difference $(p<0.05)$ within and between the groups for both the measures at the end of the 3rd and 6th sessions. Thus, NMTT may be beneficial in decreasing the peripheralization of symptoms and pain intensity in patients with CBPS. Conclusions: NMTT can be used as an alternative and effective treatment option for patients with CBPS.
\end{abstract}

Keywords: Cervico-brachial neuralgia; Radiating pain; Neural mobilization

\section{Introduction}

One of the most common sources of musculoskeletal symptoms is a disorder of the neck with a lifetime preva- lence of $48.6 \%$. It is ranked fourth in terms of disability in the global burden of disease report [1]. Neck pain often radiates proximally to the head and/or distally to the upper back or the upper limb. One such condition is cervico-

Received Mar 14, 2020; Revised Sep 23, 2020; Accepted Oct 7, 2020

Corresponding author: Meena Makhija

Department of Physiotherapy, Indian Spinal Injuries Centre-Institute of Rehabilitation Sciences, Vasant Kunj, New Delhi, India

Tel: +91-9910011816, Fax: +91-26898810, E-mail: makhijameena81@gmail.com 
brachial pain syndrome (CBPS), defined as upper quarter pain with mechanosensitive neural tissue as the primary feature [2-5]. This condition is caused by abnormal mechanical tension in certain areas of the peripheral nervous system that results in abnormal impulse-generating sites. These sites are responsible for increased sensitivity when mechanical stress is applied along the length of the nerve and is termed mechanosensitivity [6].

CBPS is characterized by the presence of hyperexcitable features, such as pain, paresthesia, allodynia, hyperalgesia, spasm, and absence of features of neurological deficits, such as numbness, muscle weakness, and altered deep tendon reflexes [6,7]. Patients with CBPS primarily present with pain in the neck that radiates to the upper extremity $[2,8]$. Elvey's criteria, consistent with these features, confirm the involvement of neural tissue in CBPS. The criteria include active and passive movement dysfunction, adverse responses to neural tests, hyperalgesic responses to nerve root palpation and related cutaneous tissue, and the evidence of a related local area of pathology $[3,4,6,9]$.

Pain and peripheralization of the symptoms in CBPS lead to active limitation of movements during activities of daily living. Over a period of time, this exerts serious effects on physical and mental functioning, leading to disability [10]. This results in a substantial burden in terms of lower productivity that increases the cost of health care [2]. Owing to these negative effects of CBPS, it is important to reduce not only the intensity of the pain but also the radiation of symptoms. This movement of the pain from the arm to the spine is called centralization. It is characterized by progressive abolishing of the symptoms in the distal to the proximal direction in response to movement. Centralization is also described as a diagnostic and treatment tool owing to its association with a lower pain level and superior functionality. As per Werneke et al. [11], the centralization of symptoms supports favorable outcomes in patients. Although centralization is considered a predictor of the treatment outcomes in low back pain [11-16], limited studies have been conducted on patients with CBPS. It is observed that C5C6 is the most affected segment in the cervical spine. The C5-C6 nerve roots provide major autonomic supply to the median nerve; thus, patients with CBPS often present with symptoms of median nerve distribution [9]. Therefore, the centralization of symptoms along the median nerve may play a pivotal role in determining the improvement in the condition of patients with CBPS after treatment.

Physiotherapy that is prescribed specifically for the cause of pain enables faster rehabilitation. Thus, the most appropriate treatment of CBPS is considered to be the one that targets the mechanosensitive neural tissue [17]. Neural mobilization (NM) includes interventions that target the altered neurodynamics by mobilizing the neural structures either indirectly (manual techniques or exercises) or directly (sliders/tensioners techniques). Among the indirect techniques, cervical lateral glide (CLG) is a passive technique that mobilizes the structures surrounding the neural tissue and is considered the treatment of choice for palliative care in patients with CBPS $[4,6,18]$.

Among the direct techniques, NM by tensioner's technique (NMTT) involves the elongation of the nerve at both the ends. NMTT causes stretching of the shortened structures, thus playing an important role in immediate hypoalgesia and intraneural circulation [19]. NMTT has depicted a positive impact in conditions, such as low back pain, lateral epicondylalgia, and carpal tunnel syndrome; however, there is limited information regarding the effect of NMTT in CBPS [18]. Few studies have used NMTT in CBPS $[5,8]$; moreover, the findings of these studies are inconsistent, making it challenging to draw a definite conclusion. Moreover, none of these trials has evaluated the centralizing effects of NMTT, providing only preliminary evidence for the use of NMTT in patients with CBPS.

Thus, the main objective of the present study was to determine the centralizing effects of NMTT and its effect on pain in CBPS because, to our knowledge, these effects have not been previously assessed.

\section{Materials and Methods}

The study was designed as a randomized single-blinded controlled trial and was conducted as per the principles of the Declaration of Helsinki. Ethical clearance for the study was obtained from the Institutional Review Board of Jamia Hamdard University (approval no., 019). Study subjects were screened from the physiotherapy departments of various hospitals located in Delhi (India) who complained of unilateral pain in the upper quarter that radiated to the upper limb. The inclusion criteria were age 18-45 years, pain of 5-74 mm on the Visual Analog Scale (VAS) in the upper quarter that radiated to the upper limbs and was persistent for 2-12 weeks, satisfying Elvey's criteria with positive Upper Limb Tension Test-1 $[3,4,8]$. Patients with red flags for pathologic spinal condition (e.g., infection, tumors, osteoporosis, and spinal fracture); 
history of spinal surgery; prolapsed intervertebral disc; vertebrobasilar artery insufficiency; or hyperexcitability, such as reflex sympathetic dystrophy; any deformity; or soft tissue inflammatory condition were excluded.

After detailed assessment of the patients by the therapist, 33 patients ( 17 women and 16 men) with a mean age of $36.6 \pm 5.9$ years were enrolled who fulfilled the inclusion and exclusion criteria. The minimum calculated sample size of the study was 13 for each group, using the formula at $80 \%$ power and 1.1 effect size [20].

Informed consent was obtained from the patients after providing them a detailed explanation of the purpose and procedure of the study. Descriptive data for age, sex, height, weight, previous surgery, and medication history were obtained from the patients. Before starting the intervention, the patients underwent a brief clinical examination wherein the cervical, shoulder, elbow, and wrist areas were examined. The patients were informed that if during the study there was any increase in the pain or symptoms, they would be immediately withdrawn from the study and provided with suitable medical and physiotherapy intervention.

The patients were randomly assigned to two groups (A and $\mathrm{B}$ ) using a randomization software (version 1). Group A received NMTT along with conventional treatment (hot pack and postural advice with CLG), whereas group B received only conventional treatment. Treatment was provided to both groups 3 times a week for 2 weeks. NMTT and CLG were performed by the principal investigator who was certified for both the techniques and had 8 years of clinical experience. The order of performing NMTT and CLG in group A was randomized to prevent order effects. Measurements for all the patients in both groups for the centralization of symptoms and pain intensity were performed at baseline and then at the end of the 3rd and 6th sessions by an independent observer who was blinded

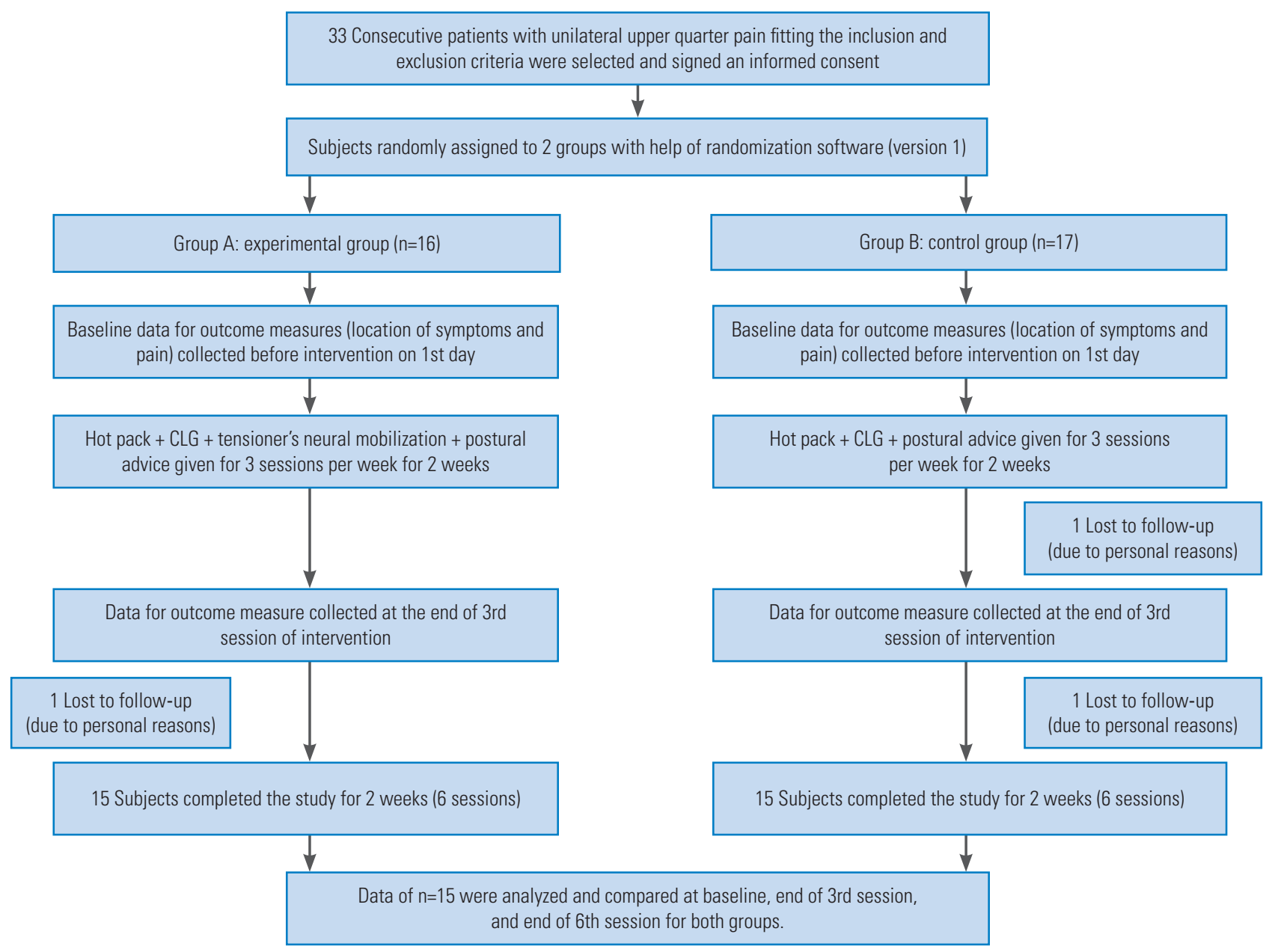

Fig. 1. Consolidated standards for reporting of trials (CONSORT) diagram. CLG, cervical lateral glide. 
to the group allocation (Fig. 1).

\section{Neural mobilization by tensioner's technique}

The patients were positioned in supine with the cervical spine in approximately $25^{\circ}$ of the contralateral lateral flexion passively by the therapist or when the first sense of increased resistance was perceived. Thereafter, the therapist performed steps of ULTT-1 till the patient reported first pain during elbow extension. As the position was assumed, 10 cycles of passive elbow flexion/extension, at a rate of approximately 6 seconds per cycle ( 3 seconds into extension and 3 seconds into flexion), were provided. While moving from elbow flexion to extension, an initial sense of resistance perceived by the therapist was used as a sign to alternate the directions. After the 10th cycle, a static hold was maintained with the elbow in extension for 10 seconds [19]. Three sets of 10 repetitions each were performed during each session [21]. Progression was performed as per the first pain reported by the patient during elbow extension [19].

\section{Cervical lateral glide}

The patients were positioned in supine with neutral cervical spine, shoulder abducted to $30^{\circ}$, and hand resting on the stomach. The therapist stood at the head end of the couch with one of her hands supporting the patient's affected shoulder over the acromial region and the other hand holding and supporting the head and neck. The radial border of the metacarpophalangeal joint of the index finger of the therapist was used to contact the patient's articular pillar of the specified segment, whereas the fourth and fifth fingers supported the patient's head. The technique involved a gentle controlled lateral glide of C5-C6 away from the involved side in a slow oscillating manner to a point in range where the first perception of resistance was experienced by the therapist (and before pain onset). This was considered as a treatment barrier [4]. Three sets of each for 30 seconds (10 oscillations) were performed per session [22]. Progression of technique was done with the shoulder in a gradually increasing range of abduction [6].

Both groups were provided a hot pack in the supine position for 10 minutes before the start of the intervention, and postural advice was given after the intervention.

\section{Outcome measures}

1) Wernicke's scale

For the measurement of centralization of pain, the patients were asked to mark their most distal extent of symptoms on the body diagram that contained a scoring grid called the Wernicke's scale. Depending on the location of the pain, the grid was marked from 6 to 0 with 6 being the most distal location of pain in reference to the hand, five spinal symptoms referred to forearm, four spinal symptoms referred to arm, three spinal symptoms referred to shoulder area, two spinal symptoms referred to asymmetrical neck area, and one central neck pain, being most proximal location of pain and zero no pain. It showed excellent validity and inter-rater reliability $(\mathrm{k}=0.96-1.00)$ $[11,14,16,23]$.

\section{2) Visual Analog Scale}

This scale was used for measuring the pain intensity. The patients were asked to rate their current pain intensity on a horizontal, 100-mm line bounded by "no pain" on the left and "worst pain imaginable" on the right [24]. The validity of the scale was found to be $0.62-0.91$ with good test-retest reliability [25].

\section{Data analyses}

Statistical analyses were performed using the NCSS package ver. 12.0 (NCSS, Kaysville, UT, USA). Normality of the data and homogeneity of the variances were verified using the Kolmogorov-Smirnov and Levene tests, respectively. Demographic variables were compared across groups using independent samples $t$-test. A $p$-value $<0.05$ was considered to indicate statistical significance.

For centralization, non-parametric tests were used because the data obtained from this scale were ordinal. For between-group comparisons, we used the Mann-Whitney $U$-test. For within-group comparisons, we used Friedmann's analysis of variance (ANOVA). When a significant $p$-value was obtained, the Bonferroni post hoc test was applied to identify the differences. In all cases, the $p$-value was adjusted to $0.05 / 3=0.017$ for multiple comparisons.

A $2 \times 3$ mixed model of the ANOVA was used for analyzing pain intensity with treatment group (A versus B) as the between-subjects variable and time (pre, end of $3 \mathrm{rd}$ session, and end of 6th session) as within-subjects variable. In case of significant interactions, post hoc pairwise 
Table 1. Demographic description of the patients

\begin{tabular}{lccc} 
Characteristic & $\begin{array}{c}\text { Group A: } \\
\text { experimental group } \\
(\mathrm{n}=15)\end{array}$ & $\begin{array}{c}\text { Group B: } \\
\text { control group } \\
(\mathrm{n}=15)\end{array}$ & $p$-value \\
\hline Age $(\mathrm{yr})$ & $37.93 \pm 4.51$ & $37.80 \pm 6.88$ & $0.95^{*}$ \\
\hline Height $(\mathrm{cm})$ & $163.93 \pm 7.96$ & $165.27 \pm 10.99$ & $0.70^{*}$ \\
\hline Weight $(\mathrm{kg})$ & $65.13 \pm 4.54$ & $67.00 \pm 5.50$ & $0.32^{*}$ \\
\hline Duration of symptoms (wk) & $6.60 \pm 2.94$ & $6.93 \pm 3.05$ & $0.70^{*}$ \\
\hline
\end{tabular}

Values are presented as mean \pm standard deviation. Tested by independent sample $t$-test.

$p<0.05$.

comparisons were performed using Tukey-Kramer test for multiple comparisons.

\section{Results}

Of the 33 recruited subjects, 30 completed the study, and three dropped out. The subject recruitment is explained with the help of a flow chart (Fig. 1).

Independent $t$-test was used to identify any differences in the baseline characteristics, including age, height, and weight between the groups (Table 1). We found no significant differences in any demographic variables between the groups as $p$-value $>0.05$ making the groups comparable.

Pain scores were normally distributed; however, the data on centralization of pain were not normally distributed $(p<0.05)$, as per the Kolmogorov-Smirnov test. Moreover, variances were homogenous across the groups, as determined using Levene's test of homogeneity $(p>0.05)$.

\section{Centralization}

The average score of centralization was $5.6 \pm 0.507$ and $5.6 \pm 0.507$ in groups A and B, respectively. There was no significant difference in the centralization of pain at baseline between the groups. A significant difference was found in the 3rd session $(U=45, Z=-2.99, p=0.003)$ and 6th session of treatment $(U=19.5, Z=-3.93, p<001)$.

In group $A$, a significant difference was found in centralization across time (chi-square $=30$, degrees of freedom $[\mathrm{df}]=2, p<0.001$ ). Post hoc pairwise comparisons showed a significant decrease from baseline (median, 6; interquartile range $[\mathrm{IQR}], 5-6$ ) to the 3 rd session (median, 3; IQR, 2-5) and to the 6th session (median, $1 ; \mathrm{IQR}, 0-3$ ) at $p<0.001$.

In group B, there was a significant difference in cen-

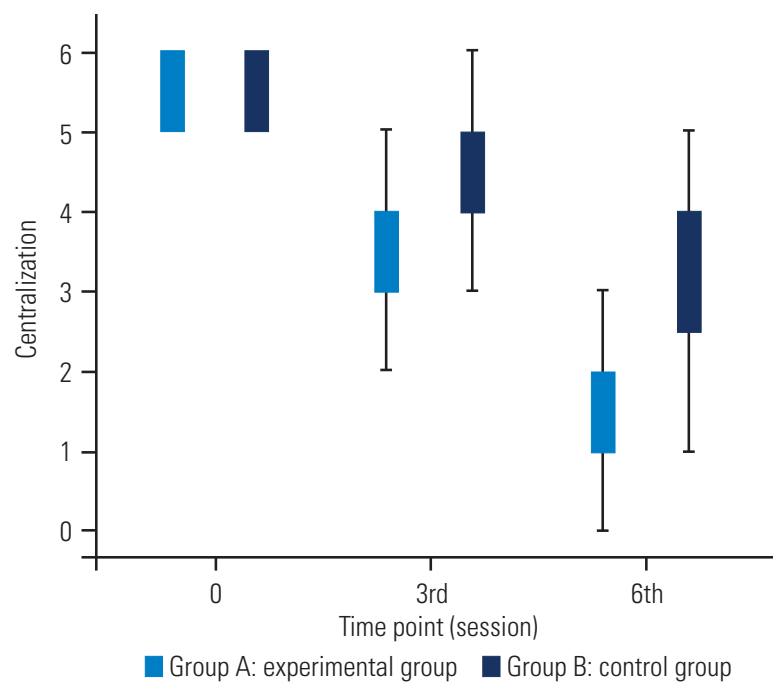

Fig. 2. Centralization scores across groups at each assessment point.

tralization across time (chi-square $=27.74, \mathrm{df}=2, p<0.001$ ). Post hoc pairwise comparisons showed a significant decrease from baseline (median, 6; IQR, 5-6) to the 3rd session (median, 4; IQR, 3-6) and to the 6th session (median, 3; IQR, 1-5) at $p<0.001$ (Fig. 2).

\section{Pain}

The mean pain score was $62.93 \pm 8.71$ and $60.67 \pm 8.29$ in groups $\mathrm{A}$ and $\mathrm{B}$, respectively. There was a significant interaction between groups and time $(F=29.75, \mathrm{df}=2$, $p<0.001)$. The main effect of group and time was also significant in group $\mathrm{A}(F=7.46, \mathrm{df}=1, p=0.011)$ and group $\mathrm{B}$ $(F=137.57, \mathrm{df}=2, p<0.001)$. Post hoc testing revealed that the experimental group had significantly lower pain scores at the 3rd and 6th sessions than the control group. The groups were not significantly different from each other at baseline. The reduction in pain scores was significant in both groups from baseline to the 3rd session (21 points in the experimental group and 8 points in the control group) and from the 3rd to 6th session (21 points in the experimental group and 7 points in the control group) (Fig. 3).

\section{Discussion}

This study aimed to evaluate the centralizing effects of NMTT in patients with CBPS. The use of NMTT was advocated by Beneciuk et al. [19] in 2009 based on their study on an asymptomatic population. This technique has also been supported by Coppieters et al. [26] in their 


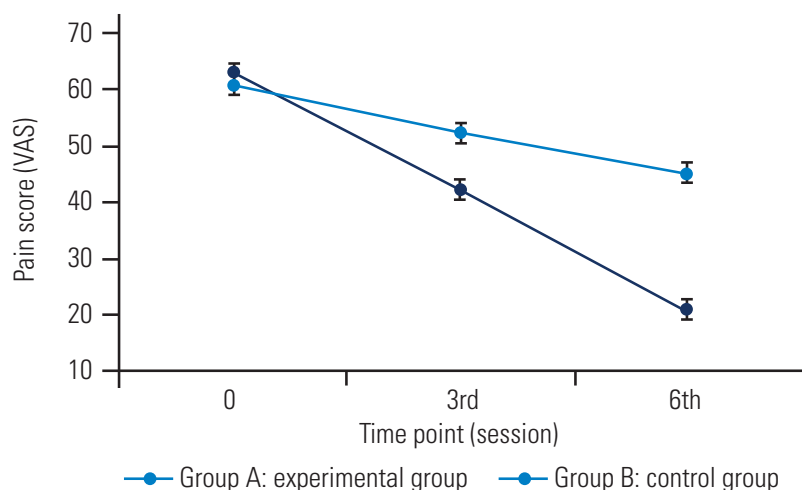

Fig. 3. Mean pain scores across groups at each assessment point. VAS, Visual Analog Scale.

2009 trial that demonstrated that the nerve excursion was greater in elbow movements with the neck in contralateral side flexion.

The results of our study demonstrate that group A was significantly better than group B in centralizing symptoms and reducing pain at both time points.

The centralization of symptoms was measured using Wernicke's scale where the scoring was done on the basis of the change in the anatomical position of pain without considering symptom intensity. This scale is standardized, valid, and reliable and has been used in several studies $[11,14,16,23]$. When the scores of centralization of symptoms were compared between the groups, a significant difference was found at the $3 \mathrm{rd}$ session $(U=45, Z=-2.99$, $p=0.003)$ and 6th session of treatment $(U=19.5, Z=-3.93$, $p<001$ ).

The possible mechanism for greater improvement in group A for the centralization of symptoms as per Shacklock can be attributed to dispersing noxious fluids by pumping action and reduction of hypoxia that, in turn, alters intraneural edema and pressure at cellular levels [7]. This physiological effect is also supported by Kumar et al. [27] based on their study in 2013 where they elaborated the importance of NM on the improvement in axoplasmic flow, neural vascularity causing increased neural connective tissue viscoelasticity and intra neural circulation. This improves the nerve conduction velocity, promoting reduced sensitivity of abnormal impulse-generating sites that are otherwise responsible for referring pain along neural structures. It is also possible that NMTT may have resulted in the centralization of symptoms via a reduction of the scar tissue that adheres to the neural tissue and its associated connective tissue [7]. The present findings are supported by Adel et al. [28] who demonstrated in 2011 the positive effect of tensioning techniques in producing centralization of symptoms in patients with low back pain. Similar centralizing effects of NM have been reported by Raval et al. [29] in 2014 in cervical radiculopathy. Moreover, the centralization of symptoms found in six treatment sessions in our study is also supported by Werneke et al. [11] who advocate additional medical evaluation if proximal change in location is not seen by the time of the 7th treatment visit, based on their 1999 study.

The tensioner's technique used in this study reproduced symptoms in few patients and lead to peripheralization. However, this peripheralization was short term and was reduced as soon as the arm was moved into a relaxed position (elbow flexion). Further, the duration for which the tensioned position was maintained was very short ( $3 \mathrm{sec}-$ onds) and was therefore not capable of producing any injury or pathology. The decision to proceed with treatment despite the peripheralization of symptoms is consistent with the treatment approach used by George [30] in 2002.

The other outcome, pain intensity, was measured using the VAS, a valid and reliable scale [24]. The reduction in pain was evident and significant in group $\mathrm{A}(F=7.46, \mathrm{df}=1$, $p=0.011)$ than in group $B(F=137.57, \mathrm{df}=2, p<0.001)$.

These results correspond to those reported by Beneciuk et al. [19] in 2009 that demonstrated an immediate hypoalgesic effect of NMTT on asymptomatic individuals. According to their study, NMTT inhibits C-fiber-mediated pain perception (temporal summation) that is generally enhanced in painful conditions. This was further supported by the studies performed by Nee and Butler [7] in 2006 and Basson et al. [18] in 2017. Studies by Basson et al. [3] in 2014 and Chandan et al. [5] in 2015 reported results similar to those obtained in our study in that NM results in significant pain relief in patients with CBPS. The methodology of these studies differed from that of our study; further, no effect on centralization was assessed. In 2008, Chhhabra et al. [8] showed NM effective in reducing pain, similar to that in our findings; however, their technique for performing NMTT differed from that used in our study.

The reduction in pain levels in both groups in our study could be attributed to the centralizing effects of NMTT because centralization is associated with less pain [14]. Furthermore, the addition of CLG and hot pack to both groups can also possibly be accounted for centralization of symptoms due to reduction of pain levels by neuro- 
physiological effects of CLG and hot pack.

The limitations of the present study were lack of followup and measurement of the association of centralization of symptoms with other outcome measures. Moreover, we relied on the patient's self-reporting of data regarding the regular consumption of medicines because they were asked to refrain from taking painkillers during the study. Future studies should compare the NMTT with sliders technique in order to identify which technique is more beneficial to patients with CBPS.

\section{Conclusions}

Overall, it can be concluded that NMTT is effective in improving the centralization of symptoms and reducing pain in CBPS, evidenced by the significant difference between the groups at 2 weeks.

\section{Conflict of Interest}

No potential conflict of interest relevant to this article was reported.

\section{Acknowledgments}

Special thanks to Dr Vandana Phadke, Scientist-D, Indian Spinal Injuries Centre, New Delhi for the statistical help and writing the results section.

\section{References}

1. Hoy D, March L, Woolf A, et al. The global burden of neck pain: estimates from the global burden of disease 2010 study. Ann Rheum Dis 2014;73:1309-15.

2. Salt E, Wright C, Kelly S, Dean A. A systematic literature review on the effectiveness of non-invasive therapy for cervicobrachial pain. Man Ther 2011;16:5365.

3. Basson CA, Stewart A, Mudzi W. The effect of neural mobilisation on cervico-brachial pain: design of a randomised controlled trial. BMC Musculoskelet Disord 2014;15:419.

4, Allison GT, Nagy BM, Hall T. A randomized clinical trial of manual therapy for cervico-brachial pain syndrome: a pilot study. Man Ther 2002;7:95-102.

5. Chandan S, Sen S, Arfath U. Effect of cervical lateral glide over neural tissue mobilization for median nerve in case of patients with cervico-brachial pain syndrome. Int J Health Rehabil Sci 2015;4:37-48.

6. Donatelli RA, Wooden MJ E. Orthopedic physical therapy. 4 th ed. St. Louis (MO): Churchill Livingstone; 2010.

7. Nee RJ, Butler D. Management of peripheral neuropathic pain: integrating neurobiology, neurodynamics, and clinical evidence. Phys Ther Sport 2006;7:3649.

8. Chhabra D, Raja K, Ganesh B, Prabhu N. Effectiveness of neural tissue mobilization over cervical lateral glide in cervico-brachial pain syndrome: a randomized clinical trial. Indian J Physiother Occup Ther 2008;2:47-52.

9. Gangavelli R, Nair NS, Bhat AK, Solomon JM. Cervicobrachial pain: how often is it neurogenic? J Clin Diagn Res 2016;10:YC14-6.

10. Daffner SD, Hilibrand AS, Hanscom BS, Brislin BT, Vaccaro AR, Albert TJ. Impact of neck and arm pain on overall health status. Spine (Phila Pa 1976) 2003;28:2030-5.

11. Werneke M, Hart DL, Cook D. A descriptive study of the centralization phenomenon: a prospective analysis. Spine (Phila Pa 1976) 1999;24:676-83.

12. Skikic EM, Suad T. The effects of McKenzie exercises for patients with low back pain, our experience. Bosn J Basic Med Sci 2003;3:70-5.

13. Werneke M, Hart DL. Centralization phenomenon as a prognostic factor for chronic low back pain and disability. Spine (Phila Pa 1976) 2001;26:758-65.

14. Werneke MW, Hart DL, Resnik L, Stratford PW, Reyes A. Centralization: prevalence and effect on treatment outcomes using a standardized operational definition and measurement method. J Orthop Sports Phys Ther 2008;38:116-25.

15. Sufka A, Hauger B, Trenary M, et al. Centralization of low back pain and perceived functional outcome. J Orthop Sports Phys Ther 1998;27:205-12.

16. Donelson R, Silva G, Murphy K. Centralization phenomenon: its usefulness in evaluating and treating referred pain. Spine (Phila Pa 1976) 1990;15:211-3.

17. Ellis RF, Hing WA. Neural mobilization: a systematic review of randomized controlled trials with an analysis of therapeutic efficacy. J Man Manip Ther 2008;16:8-22.

18. Basson A, Olivier B, Ellis R, Coppieters M, Stewart A, Mudzi W. The effectiveness of neural mobilization 
for neuromusculoskeletal conditions: a systematic review and meta-analysis. J Orthop Sports Phys Ther 2017;47:593-615.

19. Beneciuk JM, Bishop MD, George SZ. Effects of upper extremity neural mobilization on thermal pain sensitivity: a sham-controlled study in asymptomatic participants. J Orthop Sports Phys Ther 2009;39:42838.

20. Anikwe EE, Tella BA, Aiyegbusi AI, Chukwu SC. Influence of nerve flossing technique on acute sciatica and hip range of motion. Int J Med Biomed Res 2015;4:91-9.

21. Walsh MT. Upper limb neural tension testing and mobilization: fact, fiction, and a practical approach. J Hand Ther 2005;18:241-58.

22. Maitland GD, Hengeveld E, Banks K, English K. Maitland's vertebral manipulation. 7th ed. Edinburgh: Elsevier Butterworth-Heinemann; 2005.

23. Werneke MW, Hart DL. Categorizing patients with occupational low back pain by use of the Quebec Task Force Classification system versus pain pattern classification procedures: discriminant and predictive validity. Phys Ther 2004;84:243-54.

24. Jensen MP, Chen C, Brugger AM. Interpretation of visual analog scale ratings and change scores: a reanalysis of two clinical trials of postoperative pain. J Pain 2003;4:407-14.
25. Hawker GA, Mian S, Kendzerska T, French M. Measures of adult pain: Visual Analog Scale for Pain (VAS Pain), Numeric Rating Scale for Pain (NRS Pain), McGill Pain Questionnaire (MPQ), Short-Form McGill Pain Questionnaire (SF-MPQ), Chronic Pain Grade Scale (CPGS), Short Form-36 Bodily Pain Scale (SF-36 BPS), and Measure of Intermittent and Constant Osteoarthritis Pain (ICOAP). Arthritis Care Res (Hoboken) 2011;63 Suppl 11:S240-52.

26. Coppieters MW, Hough AD, Dilley A. Different nerve-gliding exercises induce different magnitudes of median nerve longitudinal excursion: an in vivo study using dynamic ultrasound imaging. J Orthop Sports Phys Ther 2009;39:164-71.

27. Kumar V, Goyal M, Rajendran N, Narkeesh D. Effect of neural mobilization on monosynaptic reflex: a pre test post test experimental design. Int J Physiother Res 2013;3:58-62.

28. Adel SM. Efficacy of neural mobilization in treatment of low back dysfunctions. J Am Sci 2011;7:566-73.

29. Raval VR, Babu KV, Kumar NS, Ghosh A. Effect of simultaneous application of cervical traction and neural mobilization for subjects with unilateral cervical radiculopathy. Int J Physiother 2014;1:269-78.

30. George SZ. Characteristics of patients with lower extremity symptoms treated with slump stretching: a case series. J Orthop Sports Phys Ther 2002;32:391-8. 